\title{
High Frequency Radio Waves - an Innovative Approach to the Correction of Age-Related Skin Changes: Clinical and mm monohistochemical Research
}

DOI: $10.17691 /$ stm2016.8.1.14

Received May 9, 2015

V.D. Trufanov, Assistant, Department of Maxillofacial Surgery and Surgical Dentistry';

E.A. Kogan, MD, DSc, Professor, Head of the Academician A.I. Strukov Pathological Anatomy Department²;

Ya.A. Yutskovskaya, MD, DSC, Professor, Director ${ }^{3}$;

N.M. Faizullina, PhD, Senior Researcher, First Department of Pathological Anatomy4;

S.Y. Ivanov, MD, DSe, Professor, Head of the Department of Maxillofacial Surgery and Surgical Dentistry';

Head of the Department of Maxillaofacial Surgery and Implantology, Postgraduate Faculty ${ }^{5}$

1 Peoples Friendship University of Russia, 6 Miklukho-Maklaya St., Moscow, 117198, Russian Federation;

21.M. Sechenov First Moscow State Medical University, 8, Bldg. 2 Trubetskaya St., Moscow, 119991, Russian Federation;

${ }_{3}^{3}$ Clinic of Professor Yutskovskaya, 26, Bldg. 1 Prospect Budyonny, Moscow, 105118, Russian Federation;

${ }^{4}$ Research Center for Obstetrics, Gynecology and Perinatology named after V.I. Kulakov, Ministry of Healthcare

of the Russian Federation, 4 Akademika Oparina St., Moscow, 117997, Russian Federation;

${ }^{5}$ Nizhny Novgorod State Medical Academy, 10/1 Minin and Pozharsky Square, Nizhny Novgorod, 603005,

Russian Federation

The aim of investigation was to evaluate the efficiency of high frequency radio wave exposure $(4.0 \mathrm{MHz})$ for the correction of age-related changes of facial skin by studying aspects of its regeneration, on the basis of morphological and immunohistochemical characteristics.

Materials and Methods. The study consisted of two parts. The clinical part included an analysis of the results of high frequency radio wave facial rejuvenation (4.0 MHz) (5 procedures: on days 1, 30,60, and months 6, 12). Immunohistochemical investigation was performed on repeated punch-biopsy specimens of the skin behind the ear, before the exposure, and at 30,60,180 and 360 days after a single exposure. Collagen of types 1 and 3, elastin, Ki-67, CD34, SMA, MMP2 and TIMP1 were identified.

Results. It was found that the most evident morphological changes after high frequency radio wave exposure occurred in the deeper dermis layers and in the adjacent adipose tissue. Such remodeling of the extracellular matrix of the dermis causes an expansion of the deep dermis layers with the accumulation of collagen of types 1 and 3 with a greater proportional increase in favor of type 1 . The key anti-ageing factor of radio wave exposure is considered to be the activation of neoangiogenesis in the dermis, which occurs gradually, reaching its maximum by month 12 after a single exposure.

Key words: high frequency radio waves; age-related skin changes; remodeling of extracellular matrix.

The problem of the correction of age-related skin changes is one the key aspects of today's esthetic medicine. Along with surgical correction and various injections of hyaluronic acid and calcium hydroxyapatite derivatives, noninvasive methods of non-surgical facelifting are gaining popularity. Among non-ablative instrument techniques RF-technologies are in widespread use, being based on radio frequency exposure for remodeling and regeneration of the dermis matrix of the skin. However it has been found that those devices for RF-lifting that operate at low frequencies (less than $1.0 \mathrm{MHz}$ ) do not have the desired effect on the process of neocollagenesis [1,2]. It is only actually possible to initiate the mechanism of remodeling the dermis and sub-dermis layers with high frequency radio waves. The method of exposing human tissues to high frequency $(4.0-6.0 \mathrm{MHz}$ ) radio waves is based on the differences in the resistance of biological tissues, and depends directly on the presence of water in the tissues.
The selective heating of the skin layers, depending on the level of hydration, to temperatures higher than $44^{\circ} \mathrm{C}$ (skin surface) and $53^{\circ} \mathrm{C}$ (subcutaneous fat), causes effects of the partial denaturation and further synthesis of collagen in the dermis and the subcutaneous adipose tissue, such that this is clinically manifested in a "lifting effect" [2, 3].

There are two high frequency radio wave technologies of noninvasive face-lifting: the first is based on a pulsed action with a frequency of $6.0 \mathrm{MHz}$, while the second one is based on continuous radio wave exposure at a frequency of $4.0 \mathrm{MHz}$. While continuous radio waves at a frequency $4.0 \mathrm{MHz}$ result in endogenous heat in the subcutaneous adipose tissue, the resistance of adipose tissues being 12 times greater than that of muscular tissues (Figure 1), the pulsed exposure at a frequency of $6.0 \mathrm{MHz}$ penetrates deeper into the tissues, resulting in overheating that can cause excessive damage, for example, fibrosis and the subsequent formation of

For contacts: Vadim D. Trufanov, e-mail: trufanov_vadim@mail.ru 
Figure 1. Coefficient of resistance of moistened skin and subcutaneous adipose tissue as a result of exposure to high frequency radio waves $(4.0 \mathrm{MHz})$

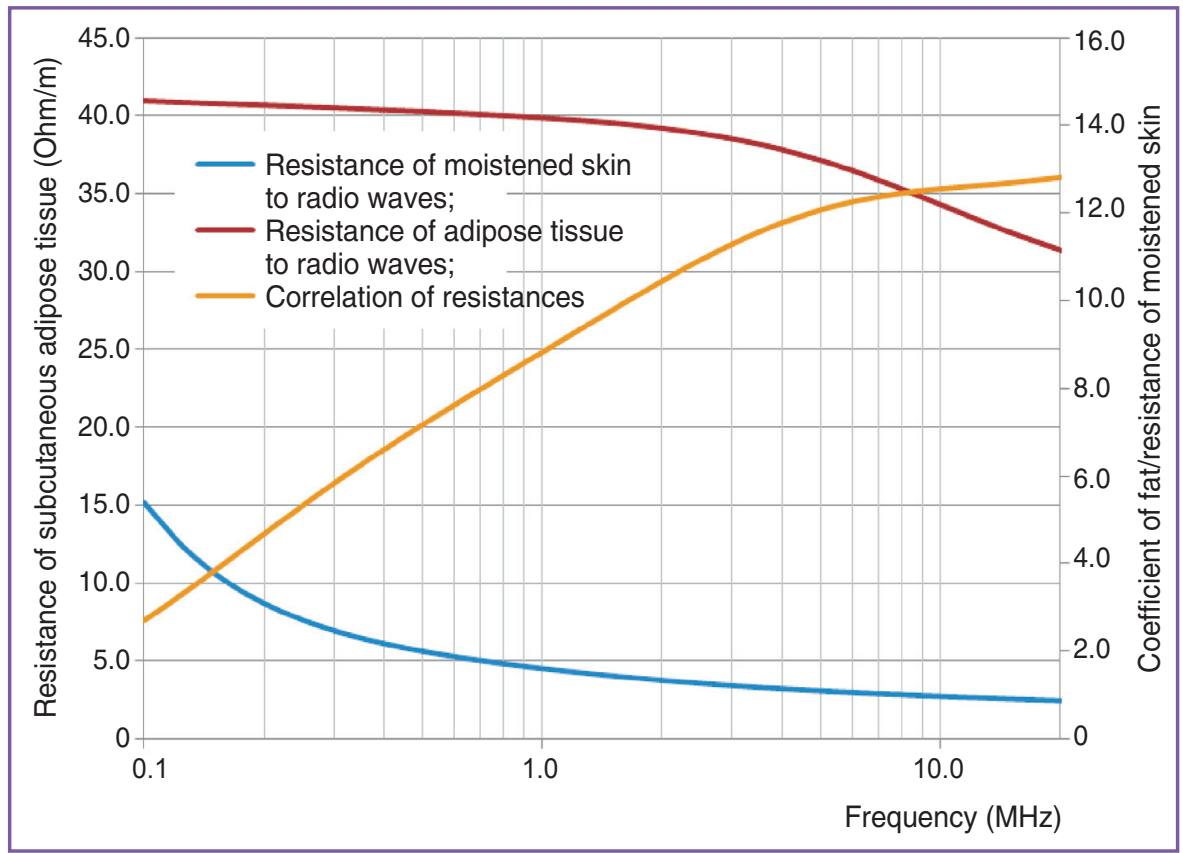

scar tissues (Figure 2). Furthermore, the continuous radio wave, to some extent, has a dual effect: firstly, it causes a partial denaturation of collagen and its further reduction; and secondly, it results in dermis microscars which are formed by microblasts, which in their turn stimulate neocollagenesis [2, 4, 5].

Continuous radio wave energy $(4.0 \mathrm{MHz})$ is widely used in surgery for dissection and for the coagulation of skin and soft tissues. As has been shown by numerous studies [3, 6, 7], it is the least disruptive method that provides for "cold" precision sectioning without charring, and allows the preservation of the underlying tissues, as well as being quick and painless and enabling a "scarless" recovery of skin wounds after surgery. The unique benefits of this technique mean that a radio wave surgical generator with a frequency of $4.0 \mathrm{MHz}$ can be successfully used for non-invasive rejuvenation and the treatment of wrinkles of degree I and II in patients with a I-VI phototype of the face and neck skin, and for the lifting of sagged and loose skin in various other parts of the body [8-10].

Previously $[3,6]$ we had demonstrated experimentally that the use of a high frequency radio wave scalpel is characterized by the quickest and most complete recovery of tissues compared to other physical methods of dissection. In the exposure area there is an intensification of neoangiogenesis of granulation tissue, with the performance of a wound defect with further epithelization and the complete recovery of the skin wound according to a restitution mechanism.

The results of pilot studies $[3,6]$ on the use of radio wave exposure for cosmetic purposes, and for achieving a rejuvenation effect, provided ideas on how exposure can stimulate neocollagenesis and the production of elastin in the dermis, in addition to neoangiogenesis, resulting in an enhancement of blood circulation. It
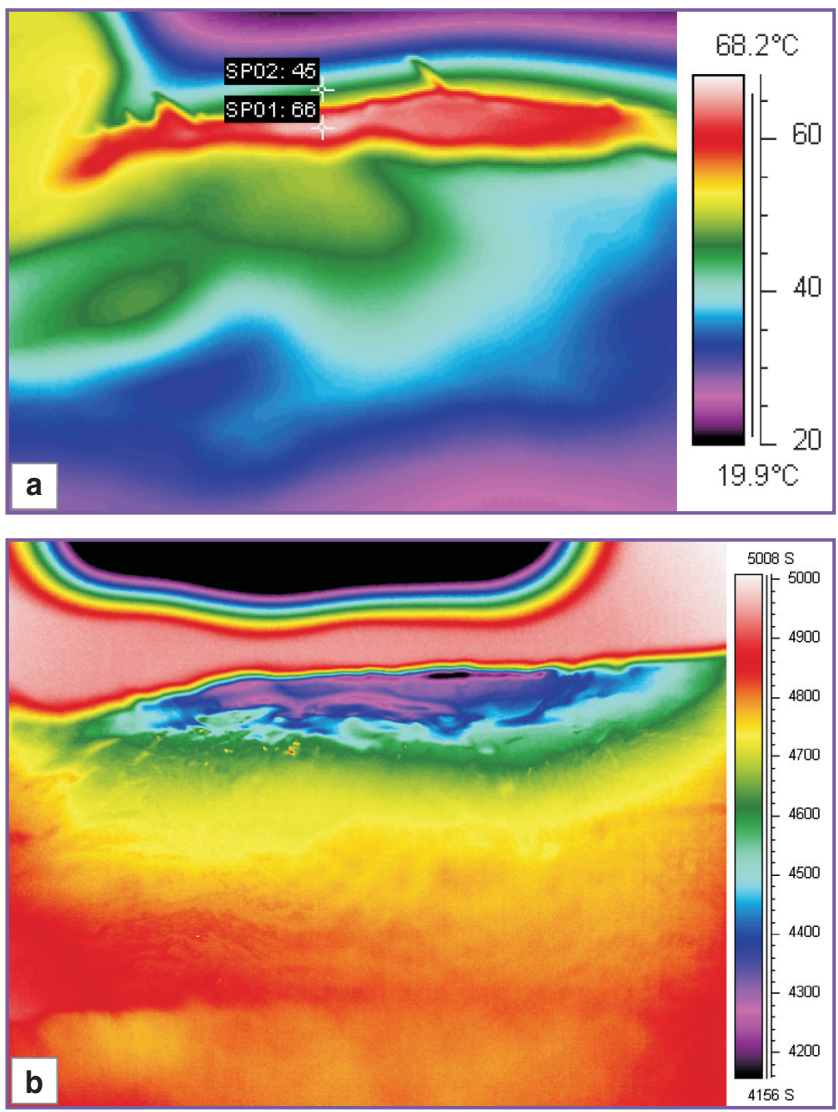

Figure 2. Thermograms of the procedures of skin exposure to: (a) continuous radio waves $(4.0 \mathrm{MHz})$; (b) pulsed at a frequency of $6.0 \mathrm{MHz}$ [4]

was found that, on the third day, the processes of neoangiogenesis at the wound edges correlate directly with epithelization. Nevertheless, the mechanism of 
radio wave skin correction at an immunohistochemical level has still not been fully studied, so does not allow us to assess its effect on the repair and proliferation of the dermis.

The aim of investigation was to evaluate the efficiency of high frequency radio wave exposure (4.0 MHz) for the correction of age-related changes of facial skin, by studying the features of its regeneration, based on morphological and immunohistochemical parameters.

Materials and Methods. The study involved 20 healthy female volunteers aged 35-58 (average age 45.0 \pm 2.41 ), among them being three women aged 5558. Five of the 20 women (ages 35-37) did not undergo any procedures, being the control group.

The study was performed in accordance with the Helsinki Declaration (accepted in June 1964 (Helsinki, Finland) and reviewed in October 2000 (Edinburgh, Scotland)) and approved by the Ethical Committee of the Peoples' Friendship University of Russia. Each patient signed an informed consent form.

The clinical investigation involved procedures using high frequency $(4.0 \mathrm{MHz})$ radio wave rejuvenation of the facial skin (face lifting and wrinkle treatment) to 15 patients from the experimental group. They underwent 5 procedures on the face (days 1, 30, 60 and months 6 , 12). The state of the facial skin was evaluated visually by the doctor and with instrumental diagnostics.

Ultrasound scanning of the facial skin was performed using a digital system of ultrasound visualization with a Skinscanner DUB (Taberna pro medicum, Germany). Linear sensors (applicators) of $22 \mathrm{MHz}$ with a depth of scanning of $10 \mathrm{~mm}$ were used.

Scanning of the facial skin was performed before and after the course of treatment (5 procedures). It allowed a noninvasive visualization of the patients' epidermis, dermis, subcutaneous adipose tissue, hair follicles, and skin vascular lumen.

The investigation of the characteristics of the functional state of the skin, namely elasticity (cutometry), hydration (corneometry), and fat content (sebumetry) was performed using a Device Soft Plus (Italy).

Standardized photodocumentation was performed on a Device Reveal Imager (Canfield, USA). The results of the therapy were evaluated by the physician, and by each patient, according to the GAIS scale, after both the first and last procedures.

A morphological investigation was performed on punch-biopsy specimens from the area behind the ear of 15 female volunteers of late reproductive and perimenopausal age (35-58). This group had undergone the single exposure to continuous radio waves of high frequency $(4.0 \mathrm{MHz})$ on the area behind the right ear.

Corresponding punch-biopsy specimens from the area behind the ear were investigated before the exposure (stage 1) and at 30, 60, 180 and 360 days (stages 2-5, respectively) after a single exposure. The biopsy specimens were fixed in $10 \%$ neutral formalin, and embedded in paraffin according to conventional technique.
Histological investigation of the biopsy specimens was performed using a morphometric method of analysis. In a series of paraffin sections stained with hematoxylin and eosin and van Gieson, we evaluated the structural characteristics of the epidermis, dermis, and adipose tissue, in addition to the state of the dermis microvessels, and the presence and degree of explicitness of lymphohistiocytic inflitration in the dermis. The morphometric part of the work involved measuring the thickness of the epidermis and dermis in relative units and calculating a coefficient representing the ratio of the "thickness of epidermis/thickness of dermis". Furthermore we evaluated the density of vessels in dermis and adipose tissue according to the number of capillary vessels in one field of view $(\times 400)$ calculated per 10 fields of view.

Immunohistochemical reactions were also performed on a series of paraffin sections, using unmasking antigens in the retriever, in accordance with the conventional protocol [11]. The double antibody method identified collagen of types 1 and 3 (DAKO CYTOMATION, Daco Inc., Denmark, at a dilution of 1:100), elastin, Ki-67 (marker for proliferating cells), CD34 (marker for neoangiogenesis), SMA (marker for smooth muscle and myofibroblast cells), MMP2 (matrix metal-proteinase, affecting the basal membrane) and TIMP1 (inhibitor of matrix metal-proteinases) (DAKO CYTOMATION, Daco Inc., Denmark, at a dilution of 1:100). The levels of expression in the tissues of collagen of types 1 and 3, elastin, MMP2, TIMP1, and CD34 according to the results of immunohistochemical investigation were semi-quantitatively scored (SQS) using standard techniques [12]. Where staining indicated expression of the marker in less than $20 \%$ of cells, expression was recorded as low -2 points, on staining more than $20 \%$ and less than $40 \%$ of cells - as moderate - 4 points, on staining more than $40 \%$ of cells - as high -6 points. Expression of the SMA marker, as well as of the marker of cellular proliferation, Ki-67, in the epidermis was scored by the percentage of positively stained cells.

The results were analyzed with statistical methods appropriate for small samples (Mann-Whitney, Student's test); and a Pearson's correlation analysis was performed.

\section{Results and Discussion}

Features of facial skin regeneration after 5 radio wave procedures. During the course of radio wave rejuvenation, all patients and researchers (physicians) noted positive dynamics in the state of the facial skin (skin tightening and a smoothing of wrinkles around the eyes and the nasolabial folds). The results of evaluation according to the GAIS scale at the time of the second procedure (30 days from the first treatment) showed the following: $87 \%$ of patients noticed positive dynamics in the state of the skin, physicians noted changed in $67 \%$ of the patients. When the points were distributed on the GAIS scale (based on scores from -1 to 3 ) the visible result was assessed by the researchers as $0.73 \pm 0.33$ points $(p<0.05)$, and by patients as $1.26 \pm 0.73$ points $(p<0.05)$ (Figure 3$)$. 


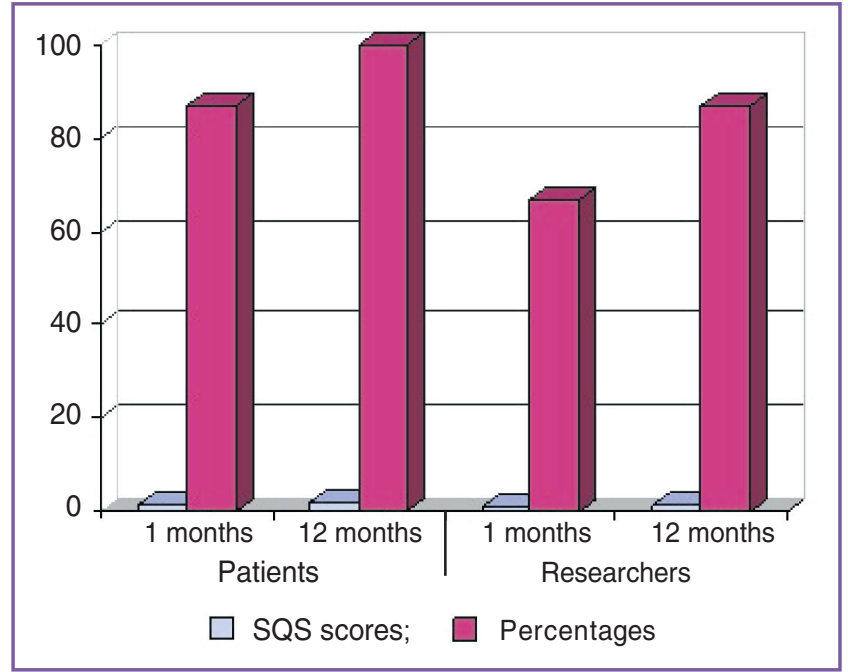

Figure 3. Subjective evaluation of patients' and researchers' satisfaction with esthetic results of the procedures in 1 and 12 months

The next evaluation of the efficacy of the therapy performed on the facial skin took place 12 months after the day of the first procedure. It revealed the following distribution: $100 \%$ of patients noted a positive effect from the procedures, their average evaluation according to the GAIS scale was $1.86 \pm 0.52$ points $(p<0.05)$; the researchers noted positive dynamics in $87 \%$ of cases, and their assessment was $1.33 \pm 0.49$ points $(p<0.05)$.

The scanning images of involutionally changed facial skin before and after procedures at a frequency of $22 \mathrm{MHz}$ provided the following picture:

1. Increase in the thickness and a leveling of the density of the epidermis were registered during the radio wave exposure. A possible reason for these observed changes is the reaction of the skin cells to the radio wave exposure and therefore a facilitation of the processes of epidermis rejuvenation. Echo-signals from the surface of the dermis were not interrupted, the inner contour of epidermis was even, and the epidermis border from the dermis was clearly defined both before and after the treatment.

2. In month 12 after the procedure of radio wave rejuvenation we could observe an increase in the average thickness of the dermis by $10.7 \%$, and a growth in the average acoustic density of the dermis by $15.3 \%$. Based on the results of the histological investigation, these facts can be explained by an increase in both the synthesis of fibrous components and in the amount of extracellular matrix, in response to an inflammatory reaction induced by the radio wave exposure. Before and after the treatment the border between the dermis and the adipose tissue was clear.

Thus, the method of high frequency ultrasonography showed an explicit locally-spread reaction to the radio wave exposure, an increase in the synthesis of fibrous components and an increase in the amount of the intercellular matrix in dermis.

The study indicated steady positive dynamics of changes in the functional characteristics of the state of the skin. According to corneometry, the change in the hydration level in relative units compared to the age norm had grown to normal values (from 41.7 to 44.4 c.u. - by $6 \%$, the norm being 44 c.u.; $p<0.05$ ). Possibly, these changes are the result of recovery of the structure and thus of the water retention capacity of the epidermis and dermis. The elasticity parameter, according to cutometry, had grown by $5 \%$ (from 39.6 to 41.6 c.u., the norm for this age group being 26 c.u.; $p<0.05)$. The cutometry findings also correlated with the ultrasound image showing an increase in echodensity of the dermis and an enhancement of its fibrous component. The fat content of the skin had increased by $4.5 \%$ (45.93-98.10 c.u., the norm being 46 c.u.; $\mathrm{p}=0.05$ ), which indicates an indirect influence of radio wave exposure on the restructuring effect, and on the level of sebum secretion.

The second part of the study was performed on material from the punch-biopsy specimens from the area behind the ear, and we evaluated the morphological and immunohistochemical manifestations of the dynamics of remodeling of the extracellular matrix of the dermis after a single radio wave exposure $(4.0 \mathrm{MHz})$.

Morphological and immunohistochemical changes in the skin in an area behind the ear at different stages of observation. The morphological features

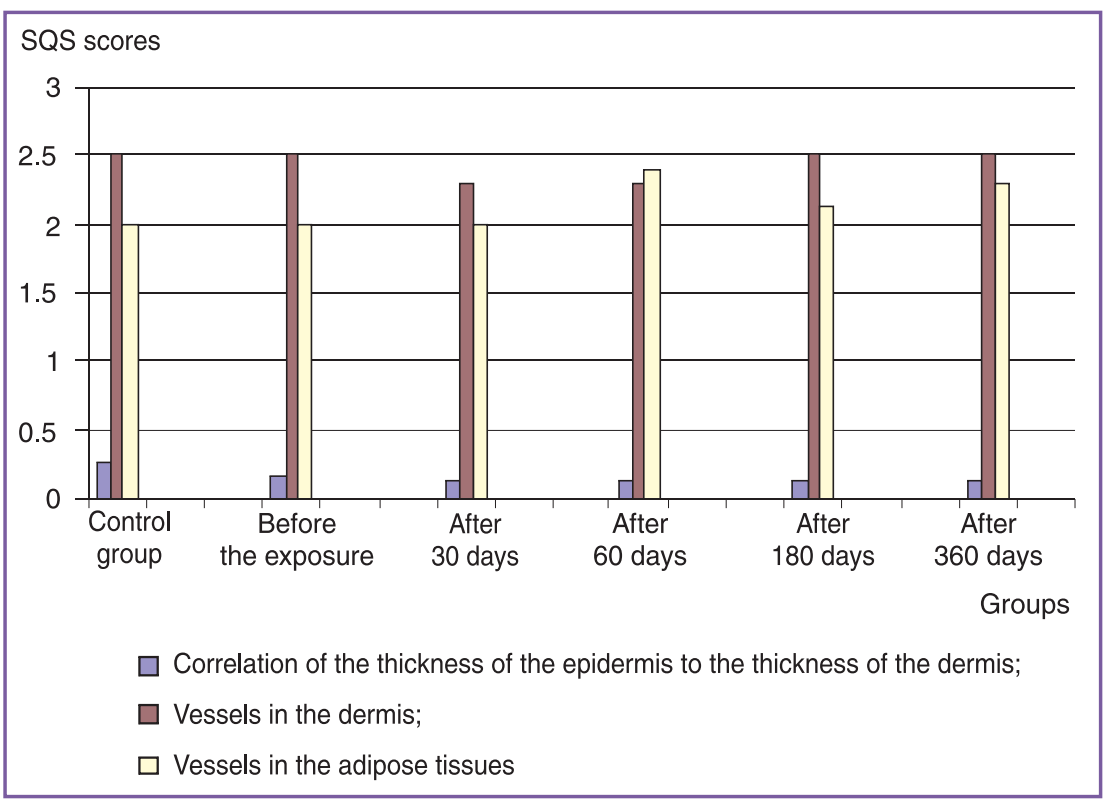

Figure 4. Morphological characteristic of female skin in the area behind the ear in the experimental and control groups at different stages of radio wave exposure 


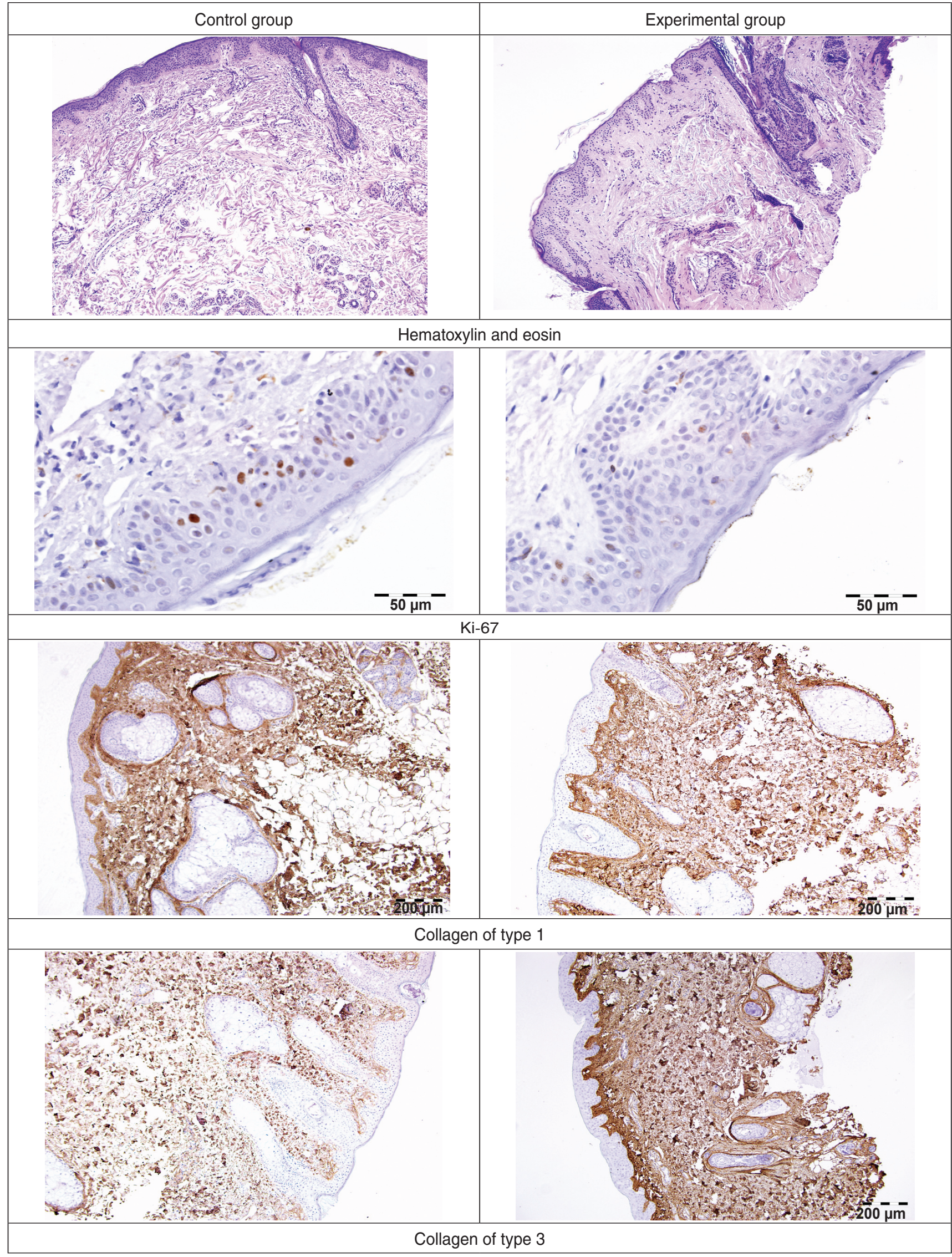

Figure 5. Structural features of female skin in the area behind the ear in the control and experimental group before radio wave exposure 


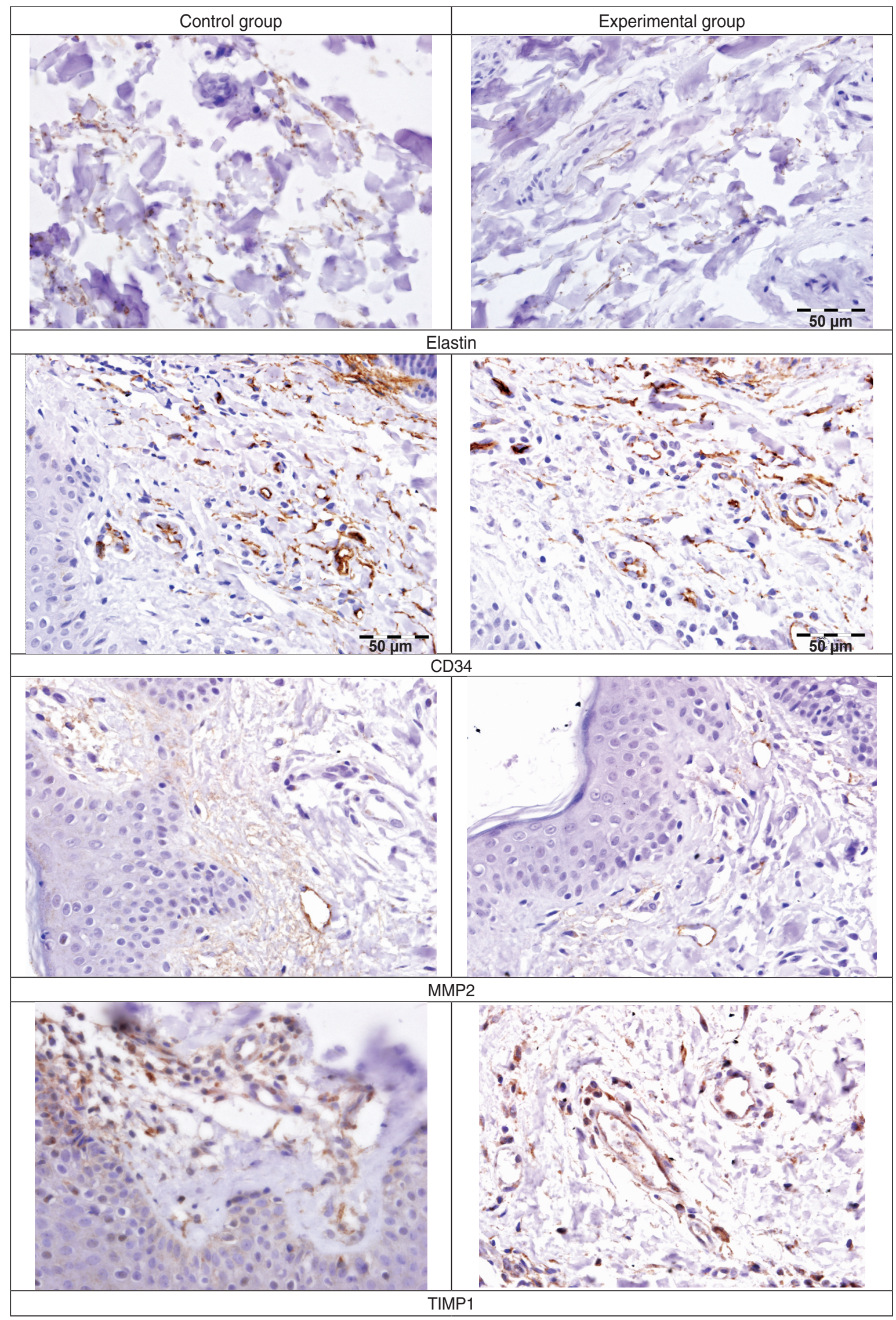

Figure 6. Immunohistochemical features of female skin in the area behind the ear in the control and experimental group before radio wave exposure 
of female skin in the area behind the ear from the experimental group (in an upper age range: 35-58) compared to female skin from the control group (aged 35-37 years) before the procedure, included a relatively thinner epidermis compared to the dermis, extending to atrophic changes in 3 out of the 15 patients (Figure 4).

Structural changes in female skin from the experimental group after radio wave exposure were manifested in 30 days after the procedure and were as follows: an enhancement of neoangiogenesis in the deep dermis layers and in the adipose tissue, as well as an increase in the relative thickness of the dermis $(p<0.05)$.

The immunohistochemical characteristics of female skin in the area behind the ear compared to the women from the control group before the radio wave exposure indicated the following features (Figures 5, 6):

1) a difference in the correlation between collagen of type 1 and collagen of type 3 due to a relative increase in the specific weight of collagen type 3 . Whereas in the control group the correlation "collagen of type 1/collagen of type 3" was 2, in the experimental group the value was $1.35(p<0.05)$;

2) a lower content of elastin in the dermis compared to the control group $(p<0.05)$;

3) fewer $\mathrm{CD}_{3} 4^{+}$endothelial cells compared to the control group $(p<0.05)$;

4) greater MMP2 expression compared to the control group $(p<0.05)$;

$5)$ fewer proliferating cells in the epidermis compared to the control group $(p<0.05)$.

The immunohistochemical changes of female skin in the area behind the ear in the experimental group after radio wave exposure were obvious at 30 days after the procedure and were manifested as an enhancement of neoangiogenesis in the deep layers of the dermis and subcutaneous adipose tissue, as well as in an increase in the relative thickness of the dermis $(p<0.05 ; r=0.5)$ (Figures 7-10):

1) a change in the correlation between collagen of type 1 and collagen of type 3 due to a relative increase in the specific weight of collagen of type 1 which normalized a year after a single exposure and had become equal to the correlation in the control group $(p<0.05 ; r=0.7)$;

2) an increase in the proportion of elastin in the deep layers of the dermis $(p<0.05)$;

3) a relative increase in the number of $\mathrm{CD} 34^{+}$cells $(p<0.05)$;

4) a relative decrease in MMP2 expression $(p<0.05$; $r=0.4$ );

5) a relative increase in TIMP1 expression $(p<0,05$; $r=0.6)$;

6) a relative increase in the number of proliferating cells in the epidermis $(p<0.05 ; r=0.6)$.

Hence, the morphological and immunohistochemical investigation of female skin in the area behind the ear after a single radio wave exposure $(4.0 \mathrm{MHz})$ indicated changes mostly in the deep layers of the dermis and in its adjacent subcutaneous adipose tissue, and this correlates with the findings of the physical effect of this type of energy, which causes heating of the tissues in this particular area [3]. At the same time, in the deep layers of dermis and the adjacent adipose tissue we can observe the activation of metabolic and regenerative processes resulting in an increase in the relative thickness of the dermis, and an enhancement of synthesis and the accumulation of different types of collagen and of elastin. Moreover there is also an expansion of the deep layers of the dermis along with an accumulation of collagen of types 1 and 3 with the bias towards type 1

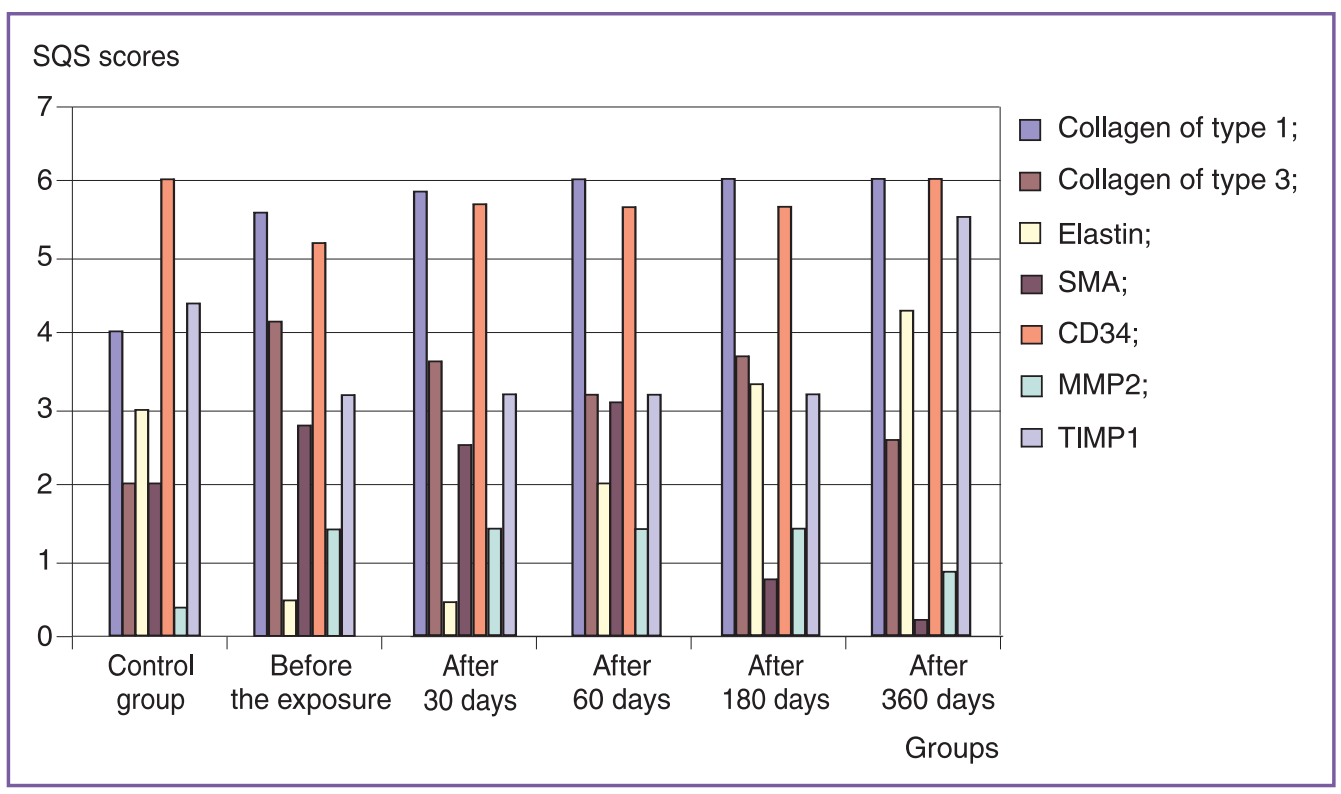

Figure 7. Immunohistochemical characteristics of female skin in the area behind the ear in the control and experimental groups at different stages of radio wave exposure 


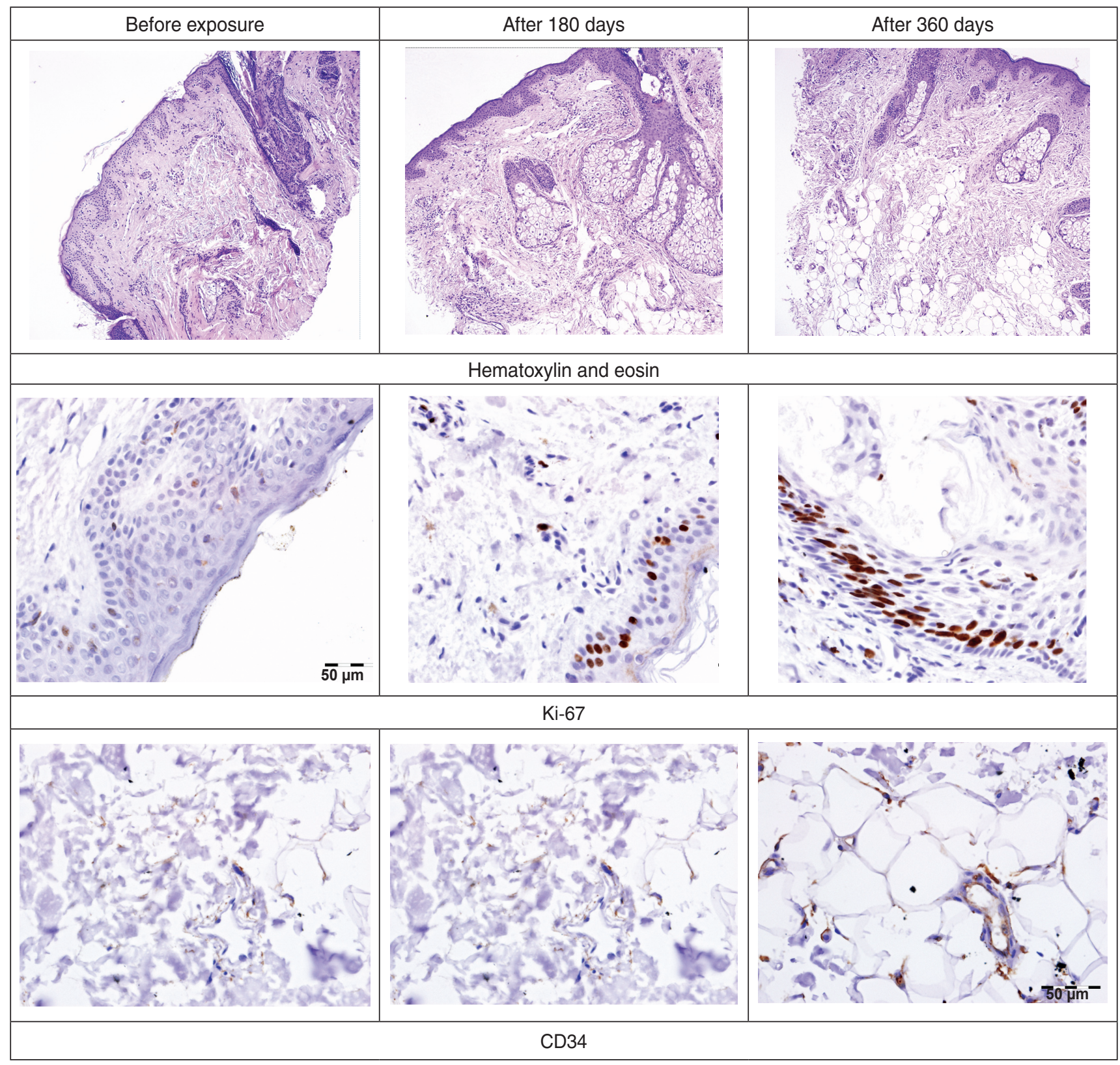

Figure 8. Structural features of female skin in the area behind the ear in the experimental group before, and at different stages after radio wave exposure. Ki-67-positive cells were evaluated in the epidermis, CD34+ cells - in the adipose tissue

being preserved, as in the women from the control group (relative norm). This process is significant for aged-related skin changes, as a decrease in the relative thickness of the dermis and decreased amounts of collagen and elastin are some of the manifestations of the ageing process. This was indicated by the findings of this study where "aged" skin was compared with the skin of young women from the control group, although the correlation of collagen of types 1 and 3 in the olderage group did not differ from that in the control group.

Another important aspect of the effect of radio waves is the enhancement of neoangiogenesis that we recorded, as it is one of the main factors causing the activation of metabolism in the tissues. It is of interest that the activation of neoangiogenesis occurred gradually, reaching its maximum by month 12 .

The third important observation from our study was the occurrence of mesenchymal cells with signs of stemminess of a myofibroblastic type (SMA-positive) in the dermis, mainly in the subcutaneous adipose tissue. Changes in the subcutaneous adipose tissue, where the number of newly formed capillaries and myofibroblast elements was increased are also interesting. The proliferative index in the surface epithelium tended to fall, compared to its value in the control group. In the deep layers of the dermis and adipose tissue the proliferative 


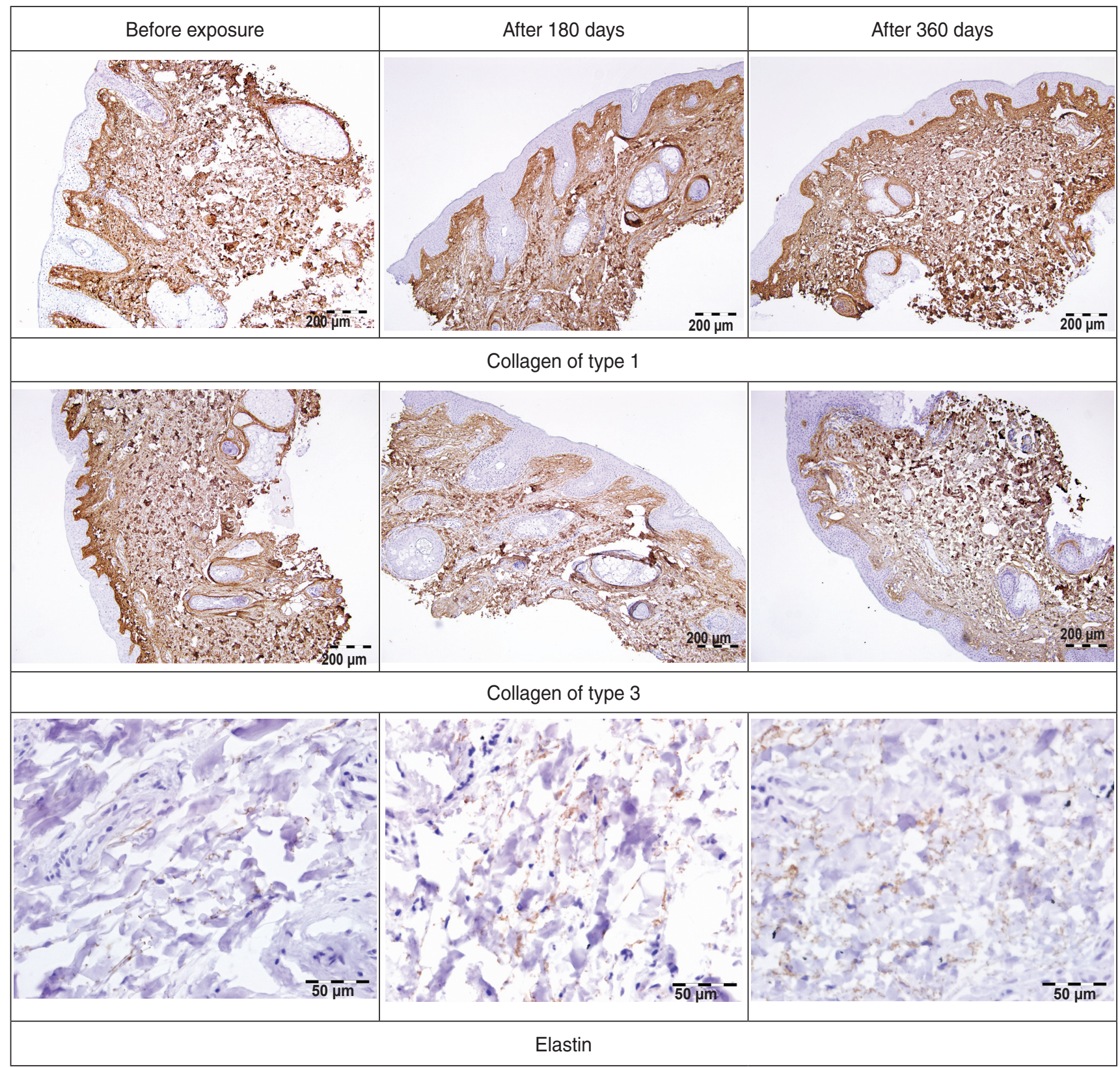

Figure 9. Components of extracellular matrix in the dermis of the area behind the ear in the experimental group before, and at different stages after radio wave exposure

index was practically zero. The described changes were increasing by day 30 and were maintained by day 180 after a single exposure.

Conclusion. Morphological and immunohistochemical investigation of female skin in the area behind the ear showed that the effect of high frequency radio waves $(4.0 \mathrm{MHz})$ to correct age-related changes is seen in the deep layers of the dermis and adjacent adipose tissue.

Remodeling of the extracellular matrix from such exposure causes expansion of the deep layers of the dermis with an accumulation of collagen of types 1 and
3 while their ratio is maintained in favor of collagen of type 1, as is seen in the dermis of young women.

The key anti-ageing factor of radio wave exposure is considered to be the activation of neoangiogenesis in the dermis which occurs gradually, reaching its maximum by month 12 after a single exposure.

One of manifestation of the anti-ageing effect of radio wave energy is the occurrence in the dermis, and in the adjacent adipose tissue mesenchymal cells of signs of stemminess of a myofibroblastic type (SMA-positive). These types of changes are involved in activation of regenerative processes. 


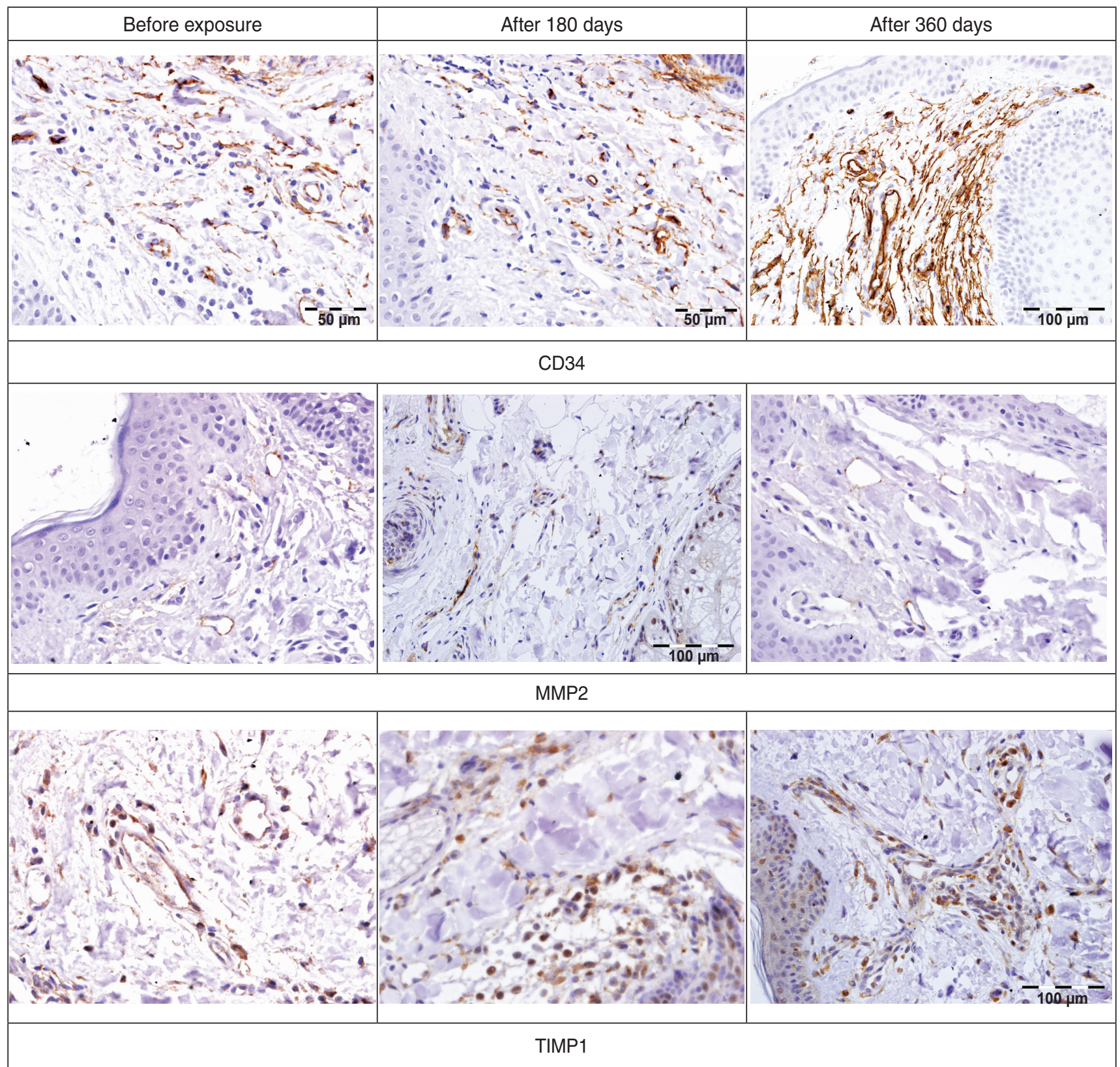

Figure 10. Comparison of expression of CD34+, MMP2, TIMP1 cells of the dermis of the patients from the experimental group before and at different stages after radio wave exposure

According to the findings of our instrumental diagnostics of facial skin after a course of radio wave exposure (5 procedures) positive dynamics could be noted in the characteristics of the skin condition.

During the ultrasound diagnostics of facial skin we recorded an explicit reaction to radio wave exposure manifested in an increase in the thickness and a levelling of the acoustic density of the epidermis, an increase in the average thickness of dermis by $10.7 \%$ and a growth in the average acoustic density of the dermis by $15.3 \%$.

According to corneometry, the level of facial skin hydration increased by $6 \%$ (reaching the norm), and the elasticity according to cutometry increased by $5 \%$. The fat content of the skin surface grew by $4.5 \%$.

The steady positive dynamics of the clinical parameters correlating with the corresponding histomorphological and immunohistochemical changes in the epidermis and dermis testify to the significance of the structural changes which occur as a result of radio wave $(4.0 \mathrm{MHz})$ exposure of the skin, and also demonstrate the "lifting-effect".

Study Funding and Conflicts of Interest. This study was not supported by any financial sources and there is no topic specific conflicts of interest related to the authors of this study. 


\section{References}

1. Belenky I., Elman M., Bar-Yosef U., Paun S.D., Vashkevich M., Levit K. Radio frequency therapy in esthetic medicine (review). Plasticheskaya khirurgiya $i$ kosmetologiya 2011(4): 705-717.

2. Lipova E.V., Glazko I.I. Physiological fundamentals of the application of "RF" and radio wave technologies in cosmetology. Apparatnaya kosmetologiya i fizioterapiya 2012; 3: 26-40.

3. Stupin V.A., Smirnova G.O., Manturova N.E., Khomyakova E.N., Kogan E.A., Polivoda M.D., Silina E.V., Sinelnikova T.G. Comparative analysis of healing processes of surgical wounds in use of various kinds of radio-frequency cutting devices and a metal scalpel. Kurskiy nauchnoprakticheskiy vestnik "Chelovek i ego zdorov'e" 2010; 4: 9-14.

4. Petrou I. RF device's steady vs. pulsed waves heat the way to less painful facial rejuvenation. Cosmetic Surgery Times 2009; 12: 28-29.

5. Rusciani A., Curinga G., Menichini G., Alfano C., Rusciani L. Nonsurgical tightening of skin laxity: a new radiofrequency approach. J Drugs Dermatol 2007; 6(4): 381386.

6. Trufanov V.D., Ivanov S.Yu., Kogan E.A., Fajzullina N.M Radiofrequency scalpels in maxillofacial and plastic surgery: experimental, immunohistochemical research. Institut stomatologii 2015; 2: 90-93.

7. Maness W.L., Roeber F.W., Clark R.E., Cataldo E., Riis D., Haddad A.W. Histologic evaluation of electrosurgery with varying frequency and waveform. J Prosthet Dent 1978; 40(3): 304-308, http://dx.doi.org/10.1016/0022-3913(78)90037-9.

8. Yutskovskaya Ya.A., Tarasenkova M.S., Kizey I.N., Trufanov V.D. Radio frequency lifting in a programme of antiageing therapy. Esteticheskaya meditsina 2010; 9(2): 3-10.

9. Yutskovskaya Ya.A., Kizey I.N., Tarasenkova M.S., Trufanov V.D. Combination of radio wave skin lifting (Pelleve) and BT-A Injections in complex programmesfor correcting involution skin changes. Esteticheskaya meditsina 2010; 9(4): 453-457.

10. Yutskovskaya Y., Kogan E., Leshunov E. A randomized, split-face, histomorphologic study comparing a volumetric calcium hydroxylapatite and a hyaluronic acid-based dermal filler. J Drugs Dermatol 2014; 13(9): 1047-1052.

11. Education guide. Immunohistochemical staining methods, fourth edition. Key M. (editor). Carpinteria, California: Dako 2006.

12. Ellinidi V.N., Anikeeva N.V. Prakticheskaya immunogistotsitokhimiya [Practical immunohistochemistry]. Saint Petersburg: VTsERM MChS Rossii; 2002. 